\title{
pH measurement system-on-foil aided with a mixed signal processor
}

\author{
Mustapha Fahem 1 , Matthias SteinmaßI', Eva-Maria Korek², Karl Neumeier ${ }^{1}$, Ralf Brederlow ${ }^{2}$, Ignaz Eisele ${ }^{1}$ \\ ${ }^{1}$ Silicon technologies and devices, Fraunhofer EMFT, Hansastr. 27D, 80797 Munich, Germany \\ ${ }^{2}$ Chair of Circuit Design, TU München, Arcisstr. 21, 80333 Munich, Germany \\ Corresponding author: matthias.steinmass/@emft.fraunhofer.de
}

\begin{abstract}
Summary:
A microcontroller with an analog front-end is integrated with 3 on-chip FG-ISFETs sensors produced at the EMFT on a pH measurement system-on-foil. The MCU offers the ability to fully control the ISFETs by keeping them at constant biasing through a software configured feedback loop. The pH sensor precision and stability are highly enhanced by reading 2 ISFETs at a time. By the differential measurement, the drift and noise compensations are achieved. The whole system can be integrated on a $10 \times 10 \mathrm{~mm}$ flexible printed circuit board.
\end{abstract}

Keywords: FG-ISFET, pH sensor, MSP430, system-on-foil, packaging

\section{Introduction}

During the last 50 years since the invention of the ion sensitive field effect transistor (ISFET) [1] many research groups proposed different configurations and techniques to realize an ISFET device on the aim of achieving stable measurements of ion concentrations, mainly $\mathrm{pH}$. An ISFET is a device where the gate electrode of a normal FET is replaced by the liquid under study. If the potential of the liquid with respect to the reference electrode changes due to a $\mathrm{pH}$ change, the threshold voltage of the FET changes accordingly. For the floating gate ISFET (FG-ISFET) a floating extended gate is pinned electrically between back-gate and reference electrode. With the introduction of the back-gate control electrode adjustment of the bias conditions for the MOSFET is possible (see fig.1). Common readout systems either set $V_{d s}$ and $I_{d}$ constant and measure gate voltage proportionally related to $\mathrm{pH}$, or measure $\mathrm{l}_{\mathrm{d}}$ at constant voltages. In this case, it is exponentially correlated to the $\mathrm{pH}$ value [2]. The encapsulation methods used with such devices have an effect on the sensor performance [3], therefore drift-free $\mathrm{pH}$ measurement requires a fluid-tight packaging which integrates also the reference electrode. The most challenging part is the packaging of the active contact area between fluid and chip [4], often done by drop casting with epoxy resins.

\section{Materials and Methods}

We present $\mathrm{pH}$ sensor chip realized at the EMFT clean room that contains 3 FG-ISFETs with different $\mathrm{pH}$ sensitivity, an on-chip reference electrode made of screen-printed $\mathrm{Ag} / \mathrm{AgCl}$ paste as well as an operational amplifier for each FGISFET to allow direct potential measurement. A simplified configuration of the electrical setup is shown in fig 1. The chip dimensions are $4.5 \times 4.5$ $\mathrm{mm}$ and the active area where the ISFETs and the reference electrode are placed is $2 \mathrm{~mm}$. The chip is packaged by a flip-chip bonding to a flexible printed circuit board (FPCB) as shown in a previous work [5]. The main objective of this work is to prove compatibility of the packaged chip and the readout realized by the Texas Instruments MSP430FR2355 using its analog I/Os and a circuit concept similar to [2]. It provides a system-on-foil, stable, drift \& noise immune $\mathrm{pH}$ measurement as well as the compatibility of the readout realized by the MCU.

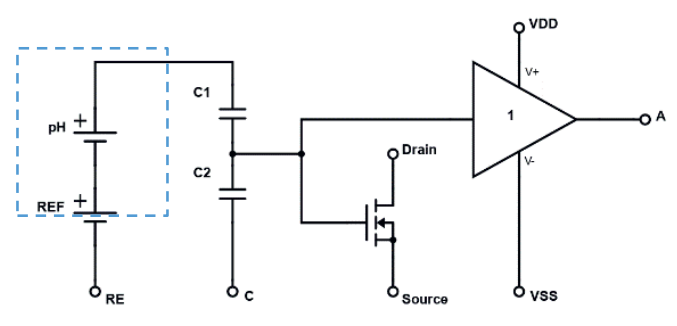

Fig. 1. One of three ISFETs featuring an on-chip opamp to monitor FG potential. The dashed blue line represents the liquid, C1\&C2 are capacitances between liquid-membrane interface and the floating gate and control electrode with floating gate respectively.

The floating gate together with the drain-source potential will set the drain current of the MOSFET. The medium potential is $\mathrm{pH}$ dependent. When it varies it will change the floating gate voltage and thus the drain current. A feedback loop needs to be implemented to control the 
biasing drain current. The working principle of this configuration is similar to the PG-ISFET described in [6]. The MSP430FR2355 offers configurable on-chip operational amplifiers and a couple of analog I/Os which make it useful for handling the readout as already shown by [2]. We keep monitoring the drain and source voltages. With the help of an external $10 \mathrm{k} \Omega$ resistor shunted with a $10 \mathrm{nF}$ capacitor, we can measure the drain current. Our configuration contains a 2 channel 4:1 MUX to measure all of the three onchip FG-ISFETs. A key experiment in this work is the reliance on 2 ISFETs with different sensitivity what is so called FET/REFET [1] configuration where one ISFET acts as a reference to the other. By differentiating the two measured signals a more stable and noise-free signal is obtained. The governing equation between electrical and chemical forces is the Nernst equation which is defined as

$$
E=E^{0}+\frac{R T}{n F} \ln \frac{[O x]}{[R e d]} .
$$

\section{Results and discussion}

All ISFETs were conditioned in $\mathrm{pH} 7$ buffer solution for at least $15 \mathrm{~min}$. The black curve on fig. 2 represents the measured potential at the ISFET2 and the red one at the ISFET1, used as reference. From the Nernst equation, the $\mathrm{pH}$ sensitivity is $\sim 59 \mathrm{mV} / \mathrm{pH}$ at room temperature, the socalled Nernst limit. According to fig. 1 we are making use of the capacitive amplification, for ISFET1 C1/C2 2.55 and for ISFET2 C1/C2 2.73. Fig. 2 shows the output voltage versus time. Respecting the capacitive gain, the sensitivity is between 30 and $40 \mathrm{mV} / \mathrm{pH}$, which corresponds to the sub-Nernst regime. The used $\mathrm{SiN}$ membrane layer is not the best sensitive material for the purpose of $\mathrm{pH}$ measurement [1]. Fig. 3 shows the subtraction of ISFET2 minus the ISFET1. This technique reduces noise and drift which makes it very promising to rely on.

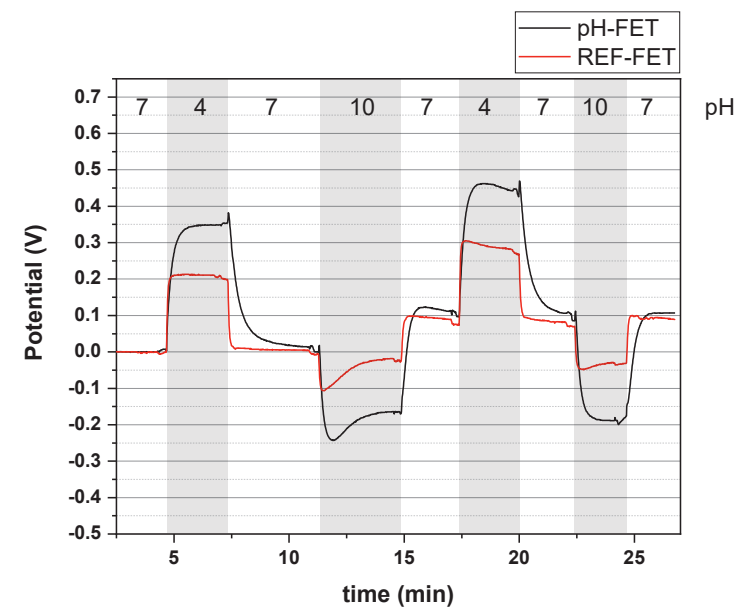

Fig. $2 \mathrm{pH}$ measurement using FET/REFET configuration. Black is the measurement of ISFET2 ( $p H-F E T)$ and red is the one of ISFET1 (REF-FET)

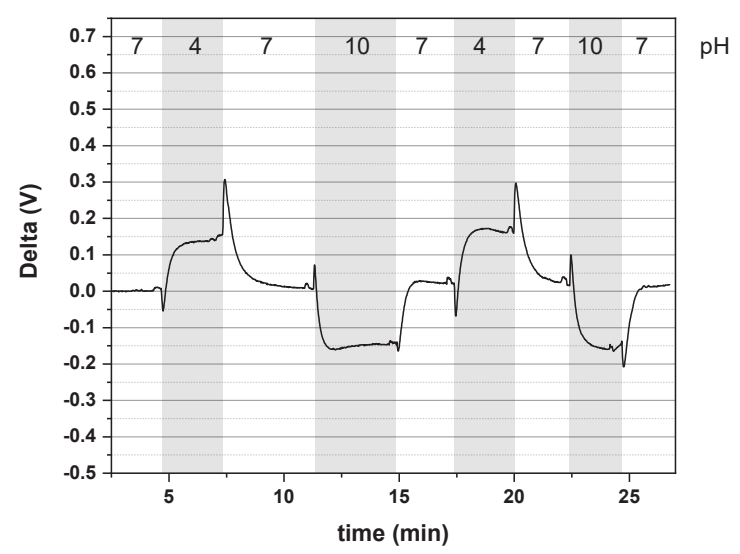

Fig.3 Differential pH measurement between FET/REFET

\section{Conclusion and Outlook}

This work shows the integration of the differential concept in a $\mathrm{pH}$ measurement system-on-foil. We used 2 ISFETs for measuring the $\mathrm{pH}$. By introducing the MCU, a $10 \times 10 \mathrm{~mm}$ system-on-foil can be established. Other ion concentration could be determined in principle by choosing the adapted sensitive membrane. After proving the concept, the next challenge would be the integration of all sensor and circuit components in a standalone easy to use system. The idea of integrating the packaging of each component on a system PCB on foil is being considered and is in the process of being realized.

\section{Acknowledgement}

Part of this work was financially supported by the FhG internal program [BioPat] and by the the Federal Ministry of Education and Research [project reference numbers 16FMD01K, 16FMD02, and 16FMD03]. We thank Markus Hefele (TUM) for support on the MSP430 programming and circuitry, Waltraud Hell (EMFT) for doing the flip-chip bonding and the EMFT cleanroom to fabricate the FG-ISFET.

\section{References}

[1] P. Bergveld, "Thirty years of ISFETOLOGY," Sensors and Actuators B: Chemical, vol. 88, no. 1, pp. 1-20, 2003

[2] M. Hefele, "Integrated multipurpose analog frontend for electrochemical ISFET sensors," IEEE NEWCAS, Munich, 2019.

[3] W. Oelßner et al., "Encapsulation of ISFET sensor chips," Sensors and Actuators B: Chemical, vol. 105, no. 1, pp. 104-117, 2005

[4] T. Datta-Chaudhuri et al, "Packaging commercial CMOS chips for lab on a chip integration," Lab on a chip, vol. 14, no. 10, 2014

[5] I. Bose et al, "Low Profile Open MEMS and ASIC Packages manufactured by Flexible Hybrid Integration in a Roll-to-Roll compatible process," in ESSDERC 2018, Dresden, 2018, pp. 102-106.

[6] P. Georgiou and C. Toumazou, "CMOS-based programmable gate ISFET," Electronics Letters, vol. 44, no. 22, pp. 1289-1290, 2008 